\title{
Explosive dark matter annihilation
}

\section{Shigeki Matsumoto*}

ICRR, Univ. Tokyo

E-mail: ismatsu@icrr

Abstract: We study pair annihilation processes of dark matter (DM) in the universe, in the case that the DM is an electroweak (EW) gauge non-singlet. In the current universe, in which the DM is highly non-relativistic, the non-perturbative effect may enhance the DM annihilation cross sections, especially for that to two $\gamma$ 's, by several orders of magnitude. We also discuss sensitivities in future searches for anomalous $\gamma$ rays from the galactic center, which originate from DM annihilation.

\section{Introduction}

Determinig the nature of the dark matter (DM) in the universe is an important problem in both particle physics and cosmology. The weakly-interacting massive particle (WIMP), $\chi^{0}$, is a good candidate for DM. It acts as the cold dark matter in the structure formation of the universe. High resolution $N$-body simulations show that the cold dark matter hypothesis provides a good explantion for structure larger than about $1 \mathrm{Mpc}$ [i] Also, the WMAP measured the cosmological abundance precisely as $\Omega_{D M}=0.27 \pm 0.04[2 \overline{2}]$. We now know the gravitational properties of DM in the structure formation and the abundance and distribution on cosmological scales. The next questions concern the constituents of DM and the distribution on galactic scales.

If the $\mathrm{DM}$ is an $\mathrm{SU}(2)_{L}$ non-singlet, a pair of DM particles could annihilate into standard-model $(\mathrm{SM})$ particles with significant cross sections as electroweak-interacting massive particle (EWIMP) DM in this paper. The detection of exotic cosmic ray fluxes, such as positrons, anti-protons and $\gamma$ rays, may be a feasible technique to search for the DM particles. Because some DM candidates in supersymmetric (SUSY) models have interactions with SM particles, these annihilation processes have been studied extensively. In particular, an excess of monochromatic $\gamma$ rays due to pair annihilation would be a robust signal if observed, because the diffused $\gamma$-ray background must have a continuous energy spectrum[ [i $\left[\begin{array}{l}4 \\ ]\end{array}\right]$. Searches for exotic $\gamma$ rays from the galactic

\footnotetext{
${ }^{*}$ Speaker.
} 
center, the galactic halo, and even from extra galaxies are projects planned for in the GLAST satellite detector and the large airshower Cerenkov telescope (ACT) arrays such as CANGAROO III, HESS, MAGIC and VERITAS.

In the previous estimates, the cross sections for the EWIMP were evaluated to leading order in the perturbation. However, DM is non-relativistic (NR) in the current universe. In this case, if the EWIMP mass $m$ is much larger than the EW scale, the EWIMP wave function may be deformed under the Yukawa potentials induced by the EW gauge boson exchanges, and it may give a non-negligible effect in the annihilation processes. Furthermore, the neutral EWIMP should have a charged $\mathrm{SU}(2)_{L}$ partner, $\chi^{ \pm}$. When the EWIMP mass is larger than EW scale, their masses are almost equal, and the unsuppressed transition between the two-body states of $2 \chi^{0}$ and $\chi^{-} \chi^{+}$may play an important role in the $2 \chi^{0}$ pair annihilation.

We reevaluate the pair annihilation cross sections of the EWIMPs, for the two cases that the $\mathrm{DM}$ is a component of two $\mathrm{SU}(2)_{L}$-doublet fermions and of an $\mathrm{SU}(2)_{L}$-triplet fermion [5]. These correspond to the Higgsino-like and Wino-like DM particles in the SUSY models, respectively. The most interesting fact we find is that the annihilation cross sections to the gauge boson pairs for the $\mathrm{SU}(2)_{L}$-doublet (resp. triplet) DM experiences a zero energy resonance around $m \simeq 6($ resp.2) $\mathrm{TeV}$, whose binding energy is zero [' $6 \overline{6}$ ] under the potential. Therefore, the cross sections would be enhanced significantly in comparison with those in the perturbative estimations for $m \geq 1$ (resp.0.5) TeV. Furthermore, it is found that the cross section for $2 \chi^{0} \rightarrow 2 \gamma$, which is usually suppressed by a one-loop factor, becomes comparable to that for the other tree-level processes, such as $2 \chi^{0} \rightarrow W^{+} W^{-}$, around the resonance. This means that the mixing between the two-body states of $\chi^{-} \chi^{+}$ and $2 \chi^{0}$ is maximal under the potential. Due to the explosive enhancement of the cross sections, the $\mathrm{SU}(2)_{L}$-triplet $\mathrm{DM}$ is partially constrained by the EGRET observation of $\gamma$ rays from the galactic center, and future $\gamma$ ray searches may be sensitive to heavier EWIMP DM.

\section{Properties of EWIMP and breakdown of the leading calculation}

First, we summarize the properties of EWIMP DMs. If DM has a vector coupling to the $Z$ boson, the current bound obtained from direct DM searches through the spin-independent interaction $[\bar{i}]$ is stringent. This means that EWIMP DM should be a Majorana fermion or a real scalar if it is relatively light. Here we consider the former case for simplicity.

A simple example for the EWIMP DMs is a neutral component of an $\mathrm{SU}(2)_{L}$-triplet fermion $(T)$ whose hypercharge is zero. This corresponds to the Wino-like LSP in SUSY models. It is accompanied by a charged fermion, $\chi^{ \pm}$. While $\chi^{0}$ and $\chi^{ \pm}$are almost degenerate in mass in the $\mathrm{SU}(2)_{L}$ symmetric limit, the EW symmetry breaking caused by the Higgs field, $h$, generates the mass splitting, $\delta m$. If $\delta m$ comes from the radiative correction, we have

$$
\delta m \simeq \frac{1}{2} \alpha_{2}\left(m_{W}-c_{W}^{2} m_{Z}\right) \sim 0.18 \mathrm{MeV}
$$


for $m \gg m_{W}$ and $m_{Z}$. Here, $m_{W}$ and $m_{Z}$ are the $W$ and $Z$ boson masses, respectively, and $c_{W} \equiv \cos \theta_{W}$, where $\theta_{W}$ is the Weinberg angle. Effective higher-dimensional operators, such as $h^{4} T^{2} / \Lambda^{3}$, also contribute $\delta m$. However they are suppressed by the new particle mass scale, $\Lambda$. The thermal relic density of the DM with mass around $1.7 \mathrm{TeV}$ is consistent with the WMAP data.

Another example for EWIMP DMs is the neutral component of a pair of $\mathrm{SU}(2)_{L^{-}}$ doublet fermions $\left(D\right.$ and $\left.D^{\prime}\right)$ with hypercharges $\pm 1 / 2$. Due to the Witten $\mathrm{SU}(2)$ anomaly $\mathrm{SU}(2)_{L^{-}}$-doublets should be paired. This corresponds to the Higgsino-like LSP in the SUSY models. In this case, $\chi^{0}$ is accompanied by a neutral Majorana fermion, $\chi^{\prime 0}$, in addition to a charged Dirac fermion, $\chi^{ \pm}$. They are again degenerate in mass in the $S U(2)_{L}$ symmetric limit. The mass difference is generated by the effective operators, such as $h^{2} D^{2} / \Lambda$, via the EW symmetry breaking. The thermal relic density of the DM explains the WMAP data in the case that the mass is around $0.6 \mathrm{TeV}$.

In the current universe, DM is believed to be highly non-relativistic, as mentioned above. In this case, the perturbative pair annihilation cross sections of the EWIMP DM may exhibit bad behavior if the DM mass is larger than the weak scale. One example is the cross section for annihilation to $2 \gamma$ at leading order. The process is induced at the one-loop level, and the cross section is

$$
(\sigma v)_{1-\mathrm{loop}} \simeq c \frac{\pi \alpha^{2} \alpha_{2}^{2}}{m_{W}^{2}}
$$

here $c$ is $4(1 / 4)$ for the $\mathrm{SU}(2)_{L}$-triplet (doublet) $\mathrm{DM}$ in the $\mathrm{SU}(2)_{L}$ symmetric limit. This cross section is not suppressed by $1 / \mathrm{m}^{2}$. On the other hand, the unitarity of the theory requires

$$
\sigma v<\frac{4 \pi}{v m^{2}},
$$

thus the perturbative unitarity is violated when $m$ is sufficiently large.

\section{Non-relativistic (NR) field theory}

The NR effective theory is useful to evaluate the cross sections in the NR limit. We studied the NR effective theory for the EWIMP perturbatively in ref. [i the unitarity violation in the cross section for annihilation to $2 \gamma$ is related to the threshold singularity. In order to evaluate the cross section quantitatively, we have to calculate the cross section non-perturbatively, using the NR effective theory.

To evaluate the annihilation cross sections for heavy EWIMPs, we need to solve the EWIMP wave function under the EW potential. In this section we give formulae for evaluation of the cross sections in the $\mathrm{SU}(2)_{L}$-triplet DM case.

The NR effective Lagrangian for two-body states, $\phi_{N}(\mathbf{r})\left(\simeq 1 / 2 \chi^{0} \chi^{0}\right)$ and $\phi_{C}(\mathbf{r})(\simeq$ $\left.\chi^{-} \chi^{+}\right)$, is given by

$$
\mathcal{L}=\frac{1}{2} \boldsymbol{\Phi}^{T}(\mathbf{r})\left[\left(E+\frac{\nabla^{2}}{m}\right) \mathbf{1}-\mathbf{V}(r)+2 i \boldsymbol{\Gamma} \delta^{3}(\mathbf{r})\right] \boldsymbol{\Phi}(\mathbf{r})
$$


where $\boldsymbol{\Phi}(\mathbf{r})=\left(\phi_{C}(\mathbf{r}), \phi_{N}(\mathbf{r})\right), \mathbf{r}$ is the relative coordinate $(r=|\mathbf{r}|)$, and $E$ is the internal energy of the two-body state. The EW potential $\mathbf{V}(r)$ is

$$
\mathbf{V}(r)=\left(\begin{array}{cc}
2 \delta m-\frac{\alpha}{r}-\alpha_{2} c_{W}^{2} \frac{e^{-m_{Z} r}}{r}-\sqrt{2} \alpha_{2} \frac{e^{-m_{W} r}}{r} \\
-\sqrt{2} \alpha_{2} \frac{e^{-m_{W} r}}{r} & 0
\end{array}\right) .
$$

In this equation, we keep only $2 \delta m$ in the $(1,1)$ component in order to calculate the DM annihilation rate up to $O(\sqrt{\delta m / m})[\overline{8}] . \quad \Gamma$ is the absorptive part of the two-point functions. Note that a factor of $1 / 2(1 / \sqrt{2})$ is multiplied for $\mathbf{V}_{22}$ and $\boldsymbol{\Gamma}_{22}$ (resp., $\mathbf{V}_{12}$ and $\boldsymbol{\Gamma}_{12}$ ), because $\phi_{N}$ is a two-body state of identical particles. Thus, $\boldsymbol{\Gamma}_{22}$ (resp., $\left.\boldsymbol{\Gamma}_{11}\right)$ is the treelevel annihilation cross section multiplied by the relative velocity $v$ and $1 / 2(1)$. Since the $\mathrm{SU}(2)_{L}$-triplet DM is assumed to be a Majorana fermion, the ${ }^{1} S$-wave gauge contribution to $\boldsymbol{\Gamma}$ is relevant to the NR annihilation. Thus, we have

$$
\boldsymbol{\Gamma}=\frac{\pi \alpha_{2}^{2}}{m^{2}}\left(\begin{array}{cc}
\frac{3}{2} & \frac{1}{2 \sqrt{2}} \\
\frac{1}{2 \sqrt{2}} & 1
\end{array}\right)
$$

The annihilation cross section of $\chi^{-} \chi^{+}$or $2 \chi^{0}$ to the EW gauge boson pair can be expressed using the $2 \times 2$ Green function, $\mathbf{G}\left(\mathbf{r}, \mathbf{r}^{\prime}\right)$, which is given by

$$
\left[\left(E+\frac{\nabla^{2}}{m}\right) \mathbf{1}-\mathbf{V}(r)+2 i \boldsymbol{\Gamma} \delta^{3}(\mathbf{r})\right] \mathbf{G}\left(\mathbf{r}, \mathbf{r}^{\prime}\right)=\delta^{3}\left(\mathbf{r}-\mathbf{r}^{\prime}\right) \mathbf{1}
$$

Emplying the optical theorem, the long-distance (wave function) and the short-distance (annihilation) effects can be factorized [igi]. The annihilation cross sections to $V V^{\prime}\left(V, V^{\prime}=\right.$ $W, Z, \gamma)$ are written

$$
(\sigma v)_{V V^{\prime}}=\left.c_{i} \sum_{a b} \boldsymbol{\Gamma}_{a b}\right|_{V V^{\prime}} \times A_{a} A_{b}^{\star}, \quad A_{a}=\int d^{3} r \mathrm{e}^{-i \mathbf{k r}}\left(E+\nabla^{2} / M\right) \mathbf{G}_{a i}(\mathbf{r}, 0),
$$

where $i$ represents the initial state $\left(i=0\right.$ and \pm for $2 \chi^{0}$ and $\chi^{-} \chi^{+}$pair annihilation, respectively) and $k=\sqrt{m E}=m v / 2$. Here $c_{0}=2$ and $c_{ \pm}=1$, where $c_{0}$ is a factor needed to compensate for the symmetric factor of $\boldsymbol{\Gamma}$ and $\mathbf{V}$. The quantity $\left.\boldsymbol{\Gamma}_{a b}\right|_{V V^{\prime}}$ is the contribution to $\boldsymbol{\Gamma}_{a b}$ from the final states, $V V^{\prime}$. It is clear that if thelong-distance effect is negligible, $(\sigma v)_{V V^{\prime}}=\left.c_{i} \boldsymbol{\Gamma}_{i i}\right|_{V V^{\prime}}$.

The $S$-wave annihilation is dominant in the NR annihilation. Thus, the Green function is reduced to $\mathbf{G}\left(\mathbf{r}, \mathbf{r}^{\prime}\right)=\mathbf{g}\left(r, r^{\prime}\right) / r r^{\prime}$. Similarly to the case in one-flavor systems, we find that $\mathbf{g}\left(r, r^{\prime}\right) / r r^{\prime}$ can be expressed in terms of the independent solutions of the homogeneous part of Eq. (3.3.

$$
\mathbf{g}\left(r, r^{\prime}\right)=\frac{m}{4 \pi} \mathbf{g}_{>}(r) \mathbf{g}_{<}^{T}\left(r^{\prime}\right) \theta\left(r-r^{\prime}\right)+\frac{m}{4 \pi} \mathbf{g}_{<}(r) \mathbf{g}_{>}^{T}\left(r^{\prime}\right) \theta\left(r^{\prime}-r\right) .
$$

The solutions $\mathbf{g}_{>}(r)$ and $\mathbf{g}_{<}(r)$ are also $2 \times 2$ matrices, because $\Phi(\mathbf{r})$ has two degrees of freedom. The boundary conditions at $r=0$ are $\left.\mathbf{g}_{<}(r)\right|_{r \rightarrow 0}=\mathbf{0},\left.\mathbf{g}_{<}^{\prime}(r)\right|_{r \rightarrow 0}=\mathbf{1}$, and 
$\left.\mathbf{g}_{>}(r)\right|_{r \rightarrow 0}=\mathbf{1}$. In the following, we assume $E<2 \delta m$, so that a pair annihilation of $\chi^{0}$ does not produce an on-shell $\chi^{-} \chi^{+}$. As a result, we find

$$
\left.\mathbf{g}_{>}(r)\right|_{r \rightarrow \infty}=\left(\begin{array}{cc}
0 & 0 \\
d_{1} \mathrm{e}^{i k r} & d_{2} \mathrm{e}^{i k r}
\end{array}\right) \text {. }
$$

In this case, the $\chi^{0}$-pair annihilation cross sections are $(\sigma v)_{V V^{\prime}}=\left.c_{i} \sum_{a b} \boldsymbol{\Gamma}_{a b}\right|_{V V^{\prime}} d_{a} d_{b}^{\star}$, as expected. In order to evaluate the cross sections, it is sufficient to calculate $d$.

\section{Numerical results and zero-energy resonance}

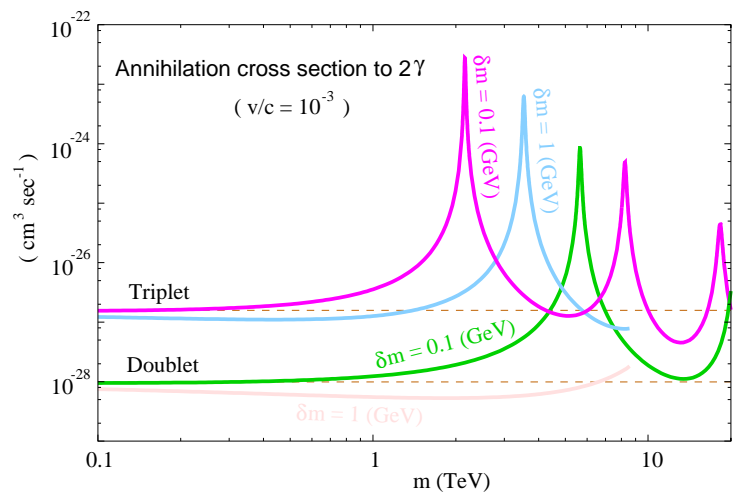

Figure 1: The $\chi^{0}$-pair annihilation cross sections to $2 \gamma$ in the case $\delta m=0.1 \mathrm{GeV}$ (solid curves) and $1 \mathrm{GeV}$ (dashed curves). Here, $\chi^{0}$ is the $\mathrm{SU}(2)_{L}$-triplet or doublet DM. In the case considered, $v / c=10^{-3}$. The leading-order cross sections in the perturbation are also plotted for $\delta m=0$ (dotted lines).

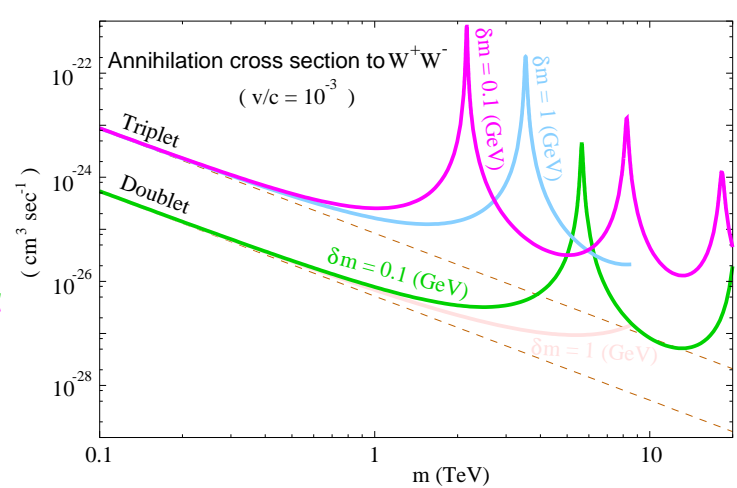

Figure 2: The $\chi^{0}$-pair annihilation cross sections to $W^{+} W^{-}$in the case $\delta m=$ $0.1 \mathrm{GeV}$ (solid curves) and $1 \mathrm{GeV}$ (dashed curves). Here, $\chi^{0}$ is the $\mathrm{SU}(2)_{L}$-triplet or doublet DM. In the case considered, $v / c=$ $10^{-3}$. The leading-order cross sections in the perturbation are also plotted for $\delta m=0$ (dotted lines).

In Fig. 自 and $\underset{2}{2}$, we display the cross sections for annihilation of the $\mathrm{SU}(2)_{L}$-triplet DM pair to $2 \gamma$ and $W^{+} W^{-}$as functions of $m$. We evaluated the cross sections numerically by using the formula in the previous section. Here, we use $v / c=10^{-3}$, which is a typical averaged velocity of DM in our galaxy, with $\delta m=0.1 \mathrm{GeV}$ and $1 \mathrm{GeV}$. The perturbative cross sections are also plotted. A large $\delta m$ leads to an unreliable numerical calculation for large $m$, and for this reason some curves are terminated at some points. However, $\delta m$ should be suppressed near these regions.

When $m$ is around $100 \mathrm{GeV}$, the cross sections for annihilation to $2 \gamma$ and $W^{+} W^{-}$ are almost the same as the perturbative ones. The cross section to $2 \gamma$ is suppressed by a loop factor in that region. However, when $m \geq 0.5 \mathrm{TeV}$, the cross sections are significantly enhanced and possess the resonance structure. In particular, the cross section to $2 \gamma$ becomes comparable to that to $W^{+} W^{-}$around the resonance. This suggests that the $2 \chi^{0}$ state is strongly mixed with $\chi^{+} \chi^{-}$. 
The qualitative behavior of the cross sections around the first resonance can be understood by approximating the EW potential by a well potential. Taking $c_{W}=1$ for simplicity, the EW potential is approximated as

$$
\mathbf{V}(r)=\left(\begin{array}{cc}
2 \delta m-b_{1} \alpha_{2} m_{W} & -b_{1} \sqrt{2} \alpha_{2} m_{W} \\
-b_{1} \sqrt{2} \alpha_{2} m_{W} & 0
\end{array}\right),
$$

for $r<R\left(\equiv\left(b_{2} m_{W}\right)^{-1}\right)$. Here, $b_{1}$ and $b_{2}$ are numerical constants. By comparing the cross sections for annihilation to $2 \gamma$ for this potential and in the case of the perturbative calculation for small $m$, we find $b_{1}=8 / 9$ and $b_{2}=2 / 3$. Under this potential, the twobody states $2 \chi^{0}$ and $\chi^{-} \chi^{+}$can form both attractive and repulsive states, whose potential energies are

$$
\lambda_{ \pm}=\frac{1}{2}\left(\mathbf{V}_{11} \pm \sqrt{\mathbf{V}_{11}^{2}+4 \mathbf{V}_{12}^{2}}\right)
$$

where $\mathbf{V}_{i j}(i, j=1,2)$ are elements in $\mathbf{V}$. The attractive state is $-\sin \theta \phi_{C}+\cos \theta \phi_{N}$, with $\tan ^{2} \theta=\lambda_{-} / \lambda_{+}$.

When $\delta m \ll b_{1} \alpha_{2} m_{W} / 2(\sim 1 \mathrm{GeV}), \theta$ is not suppressed by $\delta m$, and $\chi^{-} \chi^{+}$and $2 \chi^{0}$ are mixed under the potential. In this case, the cross section for annihilation to $2 \gamma$ is given by

$$
(\sigma v)_{2 \gamma}=\frac{4 \pi \alpha^{2}}{9 m^{2}}\left(\frac{1}{\cos \left(k_{-} R\right)}-\frac{1}{\cosh \left(k_{+} R\right)}\right)^{2},
$$

where $k_{ \pm}^{2}=\left|\lambda_{ \pm}\right| m$. Here, we ignore the $\boldsymbol{\Gamma}$ term contribution to the wave function for simplicity and take $E \simeq 0$. The cross section (4) $m_{W}$. On the other hand, it is not suppressed by the one-loop factor for $\alpha_{2} m \geq m_{W}$, and it exhibits the a correct behavior, i.e. as $\sim 1 / m^{2}$, in the large $m$ limit. When $k_{-} R=(2 n-1) \pi / 2(n=1,2, \cdots)$, the zero energy resonance, whose binding energy is zero, appears, and the cross section is enhanced significantly. In Fig. 'iili and $\overline{2}_{1}^{1}$, the $n$ th zero energy resonance appears at $m=m^{(n)} \sim n^{2} \times m^{(1)}$, while the well potential predicts $m^{(n)} \sim(2 n-1)^{2} \times m^{(1)}$. This is probably because the Yukawa potential might be approximated better by the Coulomb potential for higher zero energy resonances.

When the zero energy resonance exits, the cross sections $\sigma v$ are proportional to $v^{-2}$ for $v \ll 1$. However, this is not a signature of the breakdown of unitarity. We find from study of the one-flavor system under the well potential $V$ that when $v \ll m V \Gamma, \sigma v$ is saturated by the finite width $\Gamma$, and unitarity is not broken.

We also plot the annihilation cross sections for the $\mathrm{SU}(2)_{L}$-doublet DM in Fig. i1.i, and ${ }_{2}^{2}$. We see that the $\mathrm{SU}(2)_{L}$-doublet DM has gauge charges that are smaller than those with the $\mathrm{SU}(2)_{L}$-triplet $\mathrm{DM}$. As a result, the cross section is smaller, and the first zero energy resonance appears at $5 \mathrm{TeV}$.

\section{5. $\gamma$-ray flux from the galactic center}

The enhancement of the DM annihilation rates has significant implication for indirect searches for DM using cosmic rays. Notice that the long-distance effect is negligible for 
DM annihilation in the early universe, since the velocity at the decoupling temperature, which is typically $\sim \sqrt{1 / 5}$, is larger than $\alpha_{2}$. In the following, we discuss the search for $\gamma$ rays resulting from DM annihilation in the galactic center and future prospects.

The line $\gamma$ spectrum from pair annihilation to $2 \gamma$ or $Z \gamma$ at the galactic center is a robust signal for DM. Also, $W^{-} W^{+}$and $2 Z^{0}$ final states produce a continuum $\gamma$ spectrum due to $\pi^{0} \rightarrow 2 \gamma$, and its observation may constrain the EWIMP DM. The $\gamma$ flux, $\Psi_{\gamma}(E)$, is given by

$$
\begin{aligned}
\frac{d \Psi_{\gamma}(E)}{d E} & =9.3 \times 10^{-12}\left(\mathrm{~cm}^{-2} \mathrm{sec}^{-1} \mathrm{GeV}^{-1}\right) \times \bar{J} \Delta \Omega \\
& \times\left(\frac{100 \mathrm{GeV}}{m}\right)^{2} \sum_{V V^{\prime}} \frac{d N^{V V^{\prime}}}{d E}\left(\frac{\langle\sigma v\rangle_{V V^{\prime}}}{10^{-27}\left(\mathrm{~cm}^{3} \sec ^{-1}\right)}\right)
\end{aligned}
$$

where $N^{V V^{\prime}}$ is the number of photons from the final state $V V^{\prime}$, and $\langle\sigma v\rangle$ is the cross section averaged with respect to the velocity distribution function. Note that the angular acceptance of the detector, $\Delta \Omega$, is typically $10^{-3}$ for ACT detectors. The flux depends on the halo DM density profile $\rho$ through the reaction

$$
\bar{J} \Delta \Omega=\frac{1}{8.5 \mathrm{kpc}} \int_{\Delta \Omega} d \Omega \int_{\text {l.o.s }} d l\left(\frac{\rho}{0.3 \mathrm{GeVcm}^{-3}}\right)^{2},
$$

where the integral is along the line of sight. The DM halo $\rho$ is assumed to be spherical, $\rho=\rho(r)$, where $r^{2}=l^{2}+R_{0}^{2}-2 l R_{0} \cos \psi$ and $R_{0}$ is the solar distance to the galactic center. The quantity $\bar{J}$ has been studied for various halo models, and it is known that $3 \leq \bar{J} \leq 10^{5}$ [iㅔ] simulations, tend to give larger values of $\bar{J}$. In the following, we consider the moderate value as $\bar{J}=500$, which is typical for the NFW profile [i]

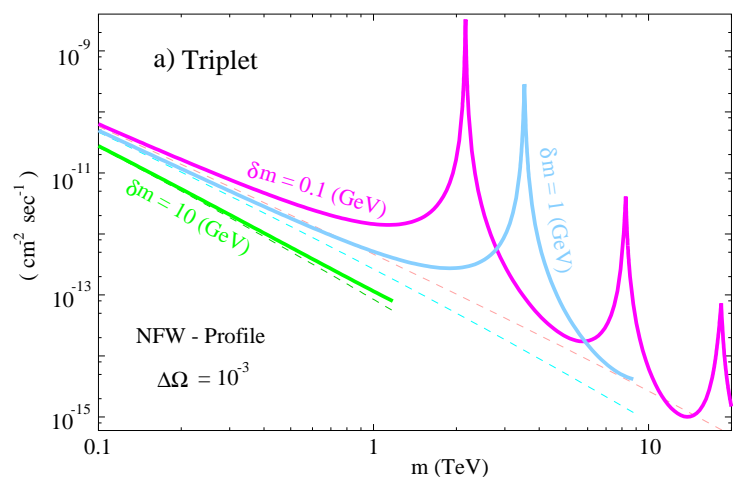

Figure 3: Line $\gamma$ flux from the galactic center in the cases of the $\mathrm{SU}(2)_{L}$-triplet $\mathrm{DM}$ particles. We take the average velocity of the $\mathrm{DM}$ as $v / c=10^{-3}, \bar{J}=500$ and $\Delta \Omega=10^{-3}$. The leading-order cross sections in the perturbation are also shown (dotted curves).

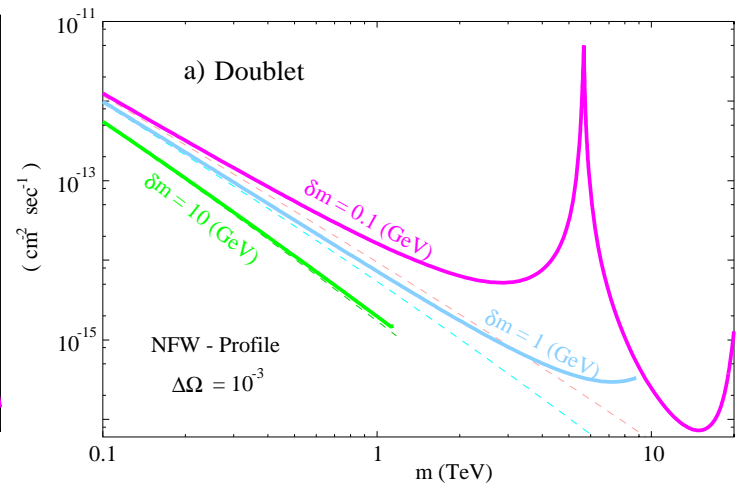

Figure 4: Line $\gamma$ flux from the galactic center in the cases of the $\mathrm{SU}(2)_{L}$-doublet $\mathrm{DM}$ particles. We take the average velocity of the $\mathrm{DM}$ as $v / c=10^{-3}, \bar{J}=500$ and $\Delta \Omega=10^{-3}$. The leading-order cross sections in the perturbation are also shown (dotted curves).

In Fig. $13 \overline{3}$, and ${ }_{-1}^{4}$, we display the line $\gamma$ flux from the galactic center in the cases of the $\mathrm{SU}(2)_{L}$-triplet and doublet $\mathrm{DM}$ particles. Here, we consider $\delta m=0.1,1,10 \mathrm{GeV}$. We 
also plot the flux obtained from the leading-order calculation for comparison. The ACT detectors have high sensitivity for $\mathrm{TeV}$-scale $\gamma$ rays. MAGIC and VERITAS in the northern hemisphere might reach to $10^{-14}\left(\mathrm{~cm}^{-2} \mathrm{~s}^{-1}\right)$ at the TeV scale while CANGAROO III and HESS in the southern hemisphere might reach $10^{-13}\left(\mathrm{~cm}^{-2} \mathrm{~s}^{-1}\right)\left[\begin{array}{l}\overline{4} \\ 1\end{array}\right]$. These ACTdetectors are expected to cover a broad region.

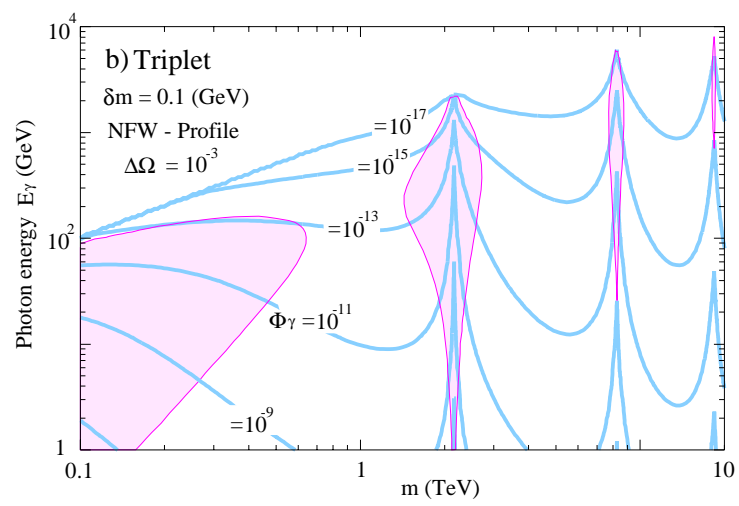

Figure 5: Contour plot of the continuum $\gamma$ ray flux from the galactic center in units of $\mathrm{cm}^{-2} \mathrm{sec}^{-1} \mathrm{GeV}^{-1}$ in the cases of the $\mathrm{SU}(2)_{L}$-triplet DM particles. Here, we use $\delta m=0.1 \mathrm{GeV}$, and the other parameters are the same as in Fig.3. The shaded regions correspond to $S / B>1$.

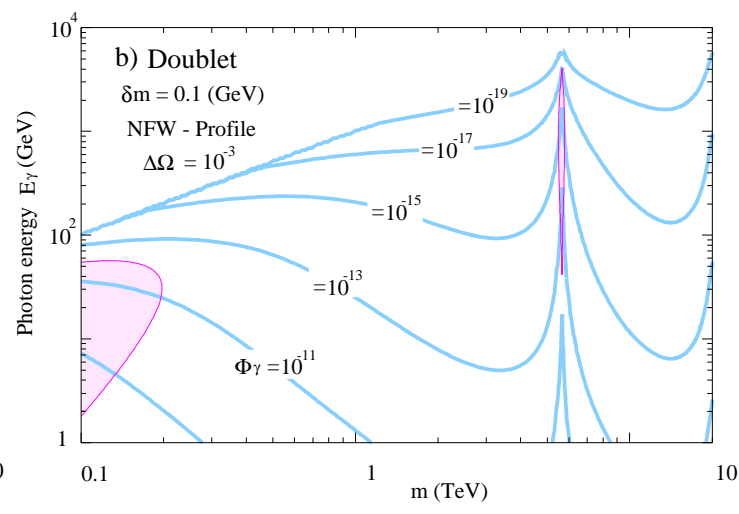

Figure 6: Contour plot of the continuum $\gamma$ ray flux from the galactic center in units of $\mathrm{cm}^{-2} \mathrm{sec}^{-1} \mathrm{GeV}^{-1}$ in the cases of the $\mathrm{SU}(2)_{L}$-doublet DM particles. Here, we use $\delta m=0.1 \mathrm{GeV}$, and the other parameters are the same as in Fig.4. The shaded regions correspond to $S / B>1$.

In Fig. $\overline{5}$ and $1 \overline{6}$, a contour plot of the continuum $\gamma$ flux from the galactic center is presented. For $d N^{V V^{\prime}} / d E$ we use the fitting functions given in Ref. [iñ regions correspond to $S / B>1$. In order to evaluate the background $B$, we assume a power law fall-off of the energy for the diffused $\gamma$ ray flux $\Psi_{B G}(E)$ in the form

$$
\frac{d \Psi_{B G}(E)}{d E}=9.1 \times 10^{-5}\left(\mathrm{~cm}^{-2} \mathrm{sec}^{-1} \mathrm{GeV}^{-1}\right) \times\left(\frac{E}{1 \mathrm{GeV}}\right)^{-2.7} \Delta \Omega .
$$

The EGRET experiment has observed diffused $\gamma$ ray emission from the galactic center up to about $10 \mathrm{GeV}$ [i] 1 in $]$. Even the small regions around the resonances, in addition to the triplet DM with $m_{\chi}=100 \mathrm{GeV}$ are constrained by the EGRET observation. The GLAST satellite detector, which should detect $\gamma$ ray with energies in the range $1 \mathrm{GeV}<E<300 \mathrm{GeV}$, has greater sensitivity in this region.

\section{References}

[1] For review, J. R. Primack, asstro-ph/02005391;

[2] D. N. Spergel et al., [astro-ph/0302209]; C. L. Bennett et al., [astro-ph/0302207].

[3] For reviews, G. Jungman, M. Kamionkowski and K. Griest, 'P hyys. Rept. 267 (1996) 195;; L. Bergstrom, Rept. Prog. Phys. 63 (2000) 793 .

[4] L. Bergstrom, P. Ullio and J. H. Buckley, Astropart. Phys. 9 91998$) 137$. 
[5] J. Hisano, S. Matsumoto and M. M. Nojiri, [hep-ph/0307216

[6] L.D. Landau, Quantum Mechanics: Non-Relativistic Theory, Volume 3, Third Edition (Elsevier Science Ltd).

[7] D. S. Akerib et al. (CDMS Collaboration), [hep-ex/03060-01]; A. Benoit et al. (the EDELWEISS Collaboration), ${ }^{\prime} \bar{h} y \bar{s}$. Lett.

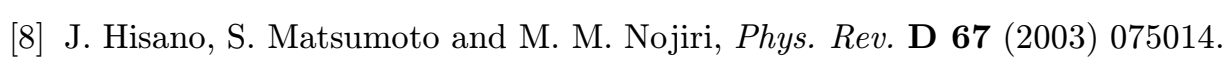

[9] G. T. Bodwin, E. Braaten and G. P. Lepage, Phhys. Rev. D 51 (1995) 1125.

[10] J.F. Navarro, C.S. Frenk, and S.D.M. Whilte, Astrophys. J. 490. $19 \overline{9} \overline{7}) 493$

[11] J.H. Hunter et al, iAstrophys. J. 481 (1997) 205 . 